\title{
Television Newscasting Analysis: Towards Establishing Language Rules in Writing News Script in Filipino for Broadcast Media
}

\section{Maria Santos-Bulaong}

Bulacan State University, Philippines, Guinhawa Street, City of Malolos, Bulacan 3000, College of Arts \& Letters- Departamento ng Araling Pilipino

\section{Abstract}

This study pertained to the grammatical errors in primetime newscasting of the two biggest network in the country, TV Patrol of ABS-CBN and 24 Oras of GMA-7. This aimed to correct the errors that were documented, believing that the one of the most popular media, Television Newscasting, will aid in teaching and enriching the Filipino

Corresponding Author: Maria Santos-Bulaong mary_bulaong@rocketmail.com

Received: 23 April 2018 Accepted: 8 May 2018 Published: 4 June 2018

Publishing services provided by Knowledge

(c) Maria Santos-Bulaong. This article is distributed under the terms of the Creative Commons Attribution License, which permits unrestricted use and redistribution provided that the original author and source are credited.

Selection and Peer-review under the responsibility of the IRCHE 2017 Conference Committee. Language because of its wide access and influence making teaching and learning process natural and with less burden. The researcher viewed the errors positively and progressively. Corder's (1971) error analysis was used to construct the descriptive taxonomy from the news stories/narratives of TV Patrol for the month of October and 24 Oras for August. For data gathering, the initial procedure was monitoring and documenting of news. The news for one month was transcribed and was kept in a data repository called data bank. Language manuals and guides were the basis in classifying the errors. The errors were analyzed and were classified into word Addition, Omission, Correct Usage, Proper Word, and Proper Word Order Category. And to ensure the accuracy of the results, the researcher used triangulation method through Personal Analysis, Focus Group Discussion and Final Discussion attended by experts in the Filipino Language and practitioners in newscasting, and from this, Filipino language rules, which can be the basis of news script writing for Broadcasting, were created.

Keywords: Television newscasting, news script, descriptive taxonomy, error analysis, language rules in Filipino,

\section{Introduction}

\section{G OPEN ACCESS}

Knowledge in grammar is of paramount importance whether it is spoken or written, and there is no exception for this, even TV news casting. There should be rules in 
language that we have to follow and know how to use them, from writing to speaking. Because of what we call grammar, relaying of message, news and information becomes clearer and more organized. Even in the listener's perspective, comprehension and interpretation of news become faster and more efficient, thanks to correct grammar usage. In short, it is important that grammar in newscasting be correct for simpler, clearer and faster relay of news and its details to the people. Also, the fact that grammar has something to do with the credibility of an individual and genre to the audience is undeniable. Newscasters are being scrutinized based on how they present news and information. If they are not careful and therefore often commit mistakes, their credibility to the people becomes weaker. It is true that grammar rules are not etched in stone, and rare mistakes are understandable; however, it should be remembered that the reputation of the writer lies on each word written on the script, and the reputation of the news reporter depends on the words coming out of his mouth. Because of Fairclough's belief (2006) that language can be planned, can be changed and can be studied, the researcher decided to study Television News Casting Analysis: Towards Establishing Language Rules in Writing News Script in Filipino for

\section{Broadcast Media.}

In the present, there is a continuous, noticeable effort of the groups, institutions and agencies of the government led by the Komisyon so Wikang Filipino (KWF) in writing and publishing language guides that strengthen the capacity of Filipino as the national language in being modernized, intellectualized and standardized despite tough competition in globalization and modern society, and these manuals are the following: Gabay sa Editing (2004, UP-SWF), Ulat na Hinggil sa Ispeling ng Wikang Filipino (2006, NCCA), Wikapedia: Balarila at Araling Filipino (KWF, 2015), Manwal na Patnubay sa Weder Forkasting (2015, PAGASA and KWF), Manwal sa Masinop na Pagsulat (2015, KWF), Patnubay so Korespondensiya Opisyal (2013, KWF), and the latest edition of Ortograpiyang Pambanso (KWF, 2014). The researcher used these manuals as the bases of analyzing transcribed broadcast news which is the corpus of the study. What is good is the distribution of these materials being done along with lecture-fora and seminar-workshops to give the teachers, professors, writers, researchers and government experts' wider and broader re-orientation about the changes in Filipino grammar. Many competitions are launched to strengthen the campaign in using those language guides. Notice that among the language guides that were mentioned above, there is no manual or language guide in writing news script in Filipino for broadcast media like TV newscasting, making it the subject- of this study. Aside from that, many tried to deviate from discussing language in broadcast media because according to them, there is a specific jargon and style in 
it, which the researcher agrees on. There is no intention of tackling style and jargon, but only grammar and the use of language to make newscasting easier and better.

\section{Objectives of the Study}

The main purpose of this study is to establish language rules based on the mistakes and common errors in grammar in regard to TV newscasting which will be the basis in establishing language rules in writing news script in Filipino for broadcast media, in line with this, the following objectives are targeted:

1. Plot primetime news believed to possess grammatical errors, coming from the two biggest TV networks in the Philippines into Descriptive Taxonomy three plane surface, which will be the main basis of the corpus of this study;

2. Analyze the grammatical errors plotted in Descriptive Taxonomy using Error Analysis;

3. Create language rules in writing news script in Filipino based on the analysis.

\section{Materials and Methods}

\subsection{Scope and delimitation}

The corpus of this study was the prime time news on 24 Oras for the month of August 2016 and IV Patrol for the month of October 2016. The focus was on the news stories/narratives that were read by the presenter inside the studio. These were transcribed and plotted in Descriptive Taxonomy; then, detection-correction was applied using Error Analysis (EA) in three columns: corpus, classification and description, and modification, but the language rules were categorized into five (5), namely:

1. Addition

2. Admission

3. Correct Usage

4. Proper Word

5. Proper Word Order 


\subsection{Methodology}

Appropriate methodology was very essential in collecting accurate data which was used in line with the objectives of this study. It encapsulated systematic processes and steps on how to administer and analyze the needed data. Descriptive - analytic methodolody and Error Analysis are used in this study.

\subsection{Research design}

Descriptive - analysis design was the overall methodology of this study. Descriptive was the most appropriate design to report and state the current phenomenon in TV Newscasting and its grammatical usage which needs analysis. In addition, analysis was needed in scrutinizing the news reports individually and determining the cause of the mistakes so that appropriate correction would be made based on language rules. In this process, inductive flow of the study transpired starting from monitoring the news, documentation of the grammatical errors, analysis, correction, and creation of language rules based on the results of the analysis.

\subsection{Theories and techniques of the study}

Corder's (1991) Error Analysis did not have any significant relationship to learning a second language as what earlier studies say. The only focus was the errors being committed in TV newscasting using the Filipino Language. The theory's viewpoint towards errors was not negative because for Error Analysis (EA), it was a chance to give correction and the effect towards studying language was constructive and progressive.

The collection and analysis of data were carefully and personally undertaken by the researcher. Triangulation was used in processing the results of the study; thus, appropriate validation made the analysis more credible. Triangulation is a technique of using more than one method to have a balanced perspective towards the study. Descriptive Taxonomy (record/list containing the parts of the news that have grammatical errors and the process to analyze those) was presented to a Focus Group Discussion composed by the faculty members from the Departamento ng Araling Pilipino of Bulacan State University using the corrective measures which means language rules were based on the collected data towards creation of rules, concepts or theories. Afterwards, FDG gave comments about the results of the analysis of Filipino linguists coming from the different universities in the country. 


\subsection{Instrumentation}

Initial interview, monitoring, transcription, and recording of the parts of the news that have grammatical errors were personally done by the researcher. Data bank was used in collecting the data each day of the study. Data bank is a modern method of keeping data with the use of a computer, and to make the data analysis better, collected information was incorporated to Descriptive Taxonomy to determine, describe, categorize, and figure out the reasons for the mistakes, correct those, and establish rules out of those. There are different types of taxonomy: comparative, descriptive, communicative-effect (Dulay, Burt, 1977 \& Krashen, 1982). Out of the three mentioned, descriptive taxonomy was used in the study; it is a record composed of columns where each steps in error analysis were undertaken. The results were presented to FGD to improve it.

\subsection{Data gathering}

Reading and asking questions initiated this study until the monitoring and transcribing of the two prime time news casting TV programs (TV Patrol and 24 Oras) for one month. Data collected for one month were thought to commit grammatical errors and were kept in the data bank. Data bank is an organized repository or a medium where information are saved and kept with the use of technology until after the data gathering procedure.

\subsection{Data processing}

From the data bank, the researcher created a Descriptive Taxonomy to plot the collected data to analyze them into three (3) steps in Error Analysis method: (1) Corpus (collection of data and recognition of error), (2) Classification \& description of errors, (3) Modification (correction). In the end, the gradual flow of the study automatically transpired - the construction of the Filipino language rules.

The contents of the Descriptive Taxonomy of Errors were given to FGD for another checking and for them to comment on it. On the other hand, the created language guide was criticized by the group of linguists came from DepEd (Dr. Anatacia Victorino), DLSU (Dr. Aurora Batnag), UST (Dr. Elimar Ravina), BSU (Dr. Victor Ramos), and one news editor from GMA-7 (Romulo Maturingan). The researcher also consulted Ron Lopez of 


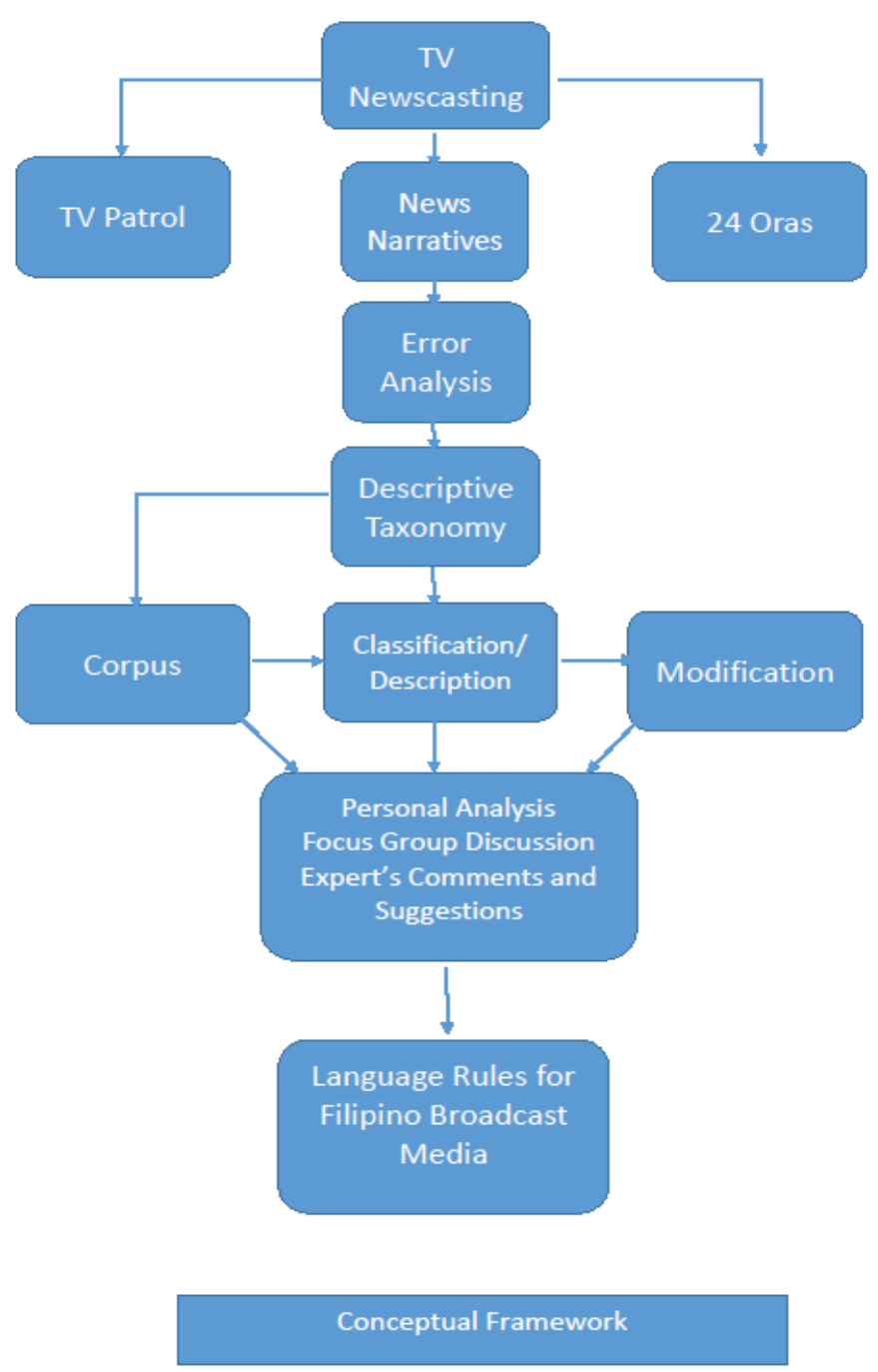

Figure 1

ABS-CBN, (Field Reporter), Dr. Galileo Zafra and Dr. Purificacion Delima of Komisyon sa Wikang Filipino for other suggestions to improve the study.

\section{Data Analysis and Discussion}

This three plane surface called Descriptive Taxonomy, is used to analyze the language error in TV news casting, 
TABLE 1: Descriptive Taxonomy.

Korpus
1. Gumagawa na sila ng GAP
para sa isang Executive Order
at nais ng DOH na mula sa
individual household na
pagpapaputok gawin itong
Community Fireworks Display.
Inaasahan nila na
mapipirmahan kaagad ang GAP
sa oras na maipasa para
maipatupad ito bago magpasko
at bagong taon at saka alam
mo sa Davao bawal ang
paputok, ang presidente ho
natin ay mayor dati ng Davao.

2. Sa mga nag-aabang ng resulta ng laban ng magkaribal na Ateneo de Manila at De La Salle University Queen Archers, nasa huling kuwarter na po ng sa iskor na eighty to fifty eight, para sa layong paralelismo. lyamado ang DLSU.

3. Kapamilya nadagdagan pa ang mga batikos ng international community sa Hitler comment ni Pangulong Duterte kabilang na po ang mga mismong Secretary of Defense ng Amerika pero ang Malacanang agad dumipensa.

4. Mayroong tatlong anak ang
biktima at nagtatrabaho po
naman sa malayong lugar ang
kanyang mister.
para sa layong paralelismo.

Binanggit ang pangalan ng Pagkakamali sa adisyon ng pahayag - isa lamang ang tinutukoy na Secretary of sakaling marami nga ang sinasabing mga secretary, pantukoy/pamparami ang nito. ay ginagamit kapag ang

\section{Deskripsyon}

Pagkakamali sa paggamit ng akronimyang di-pamilyar sa marami - Ibinabawal ang akronimya/inisyal sa pagbabalita, kung hindi ganoon katanyag ang salita/pangngalan

\section{Modipikasyon}

Gumagawa na sila ng General Advance Planning para sa isang Executive Order at nais ng DOH na mula sa individual household na pagpapaputok gawin itong Community Fireworks Display. Inaasahan nila na mapipirmahan kaagad ang GAP sa oras na maipasa para maipatupad ito bago magpasko at bagong taon.

Pagkakamali sa omisyon ng pangalan ng team ng Ateneo DLSU team, subalit nawala ang panandang pangmaramihang mga at sa pagpoposiyon nito sa Defense ng Amerika at kung dapat ay malapit o katabi ng pangngalang binibigyang turing

Pagkakamali sa paggamit ng may at mayroon. Ang mayroon sinusundang salita ay panghalip mister. na nasa anyong palagyo o kaya ay ng mga kataga o ingklitik. Kapag pangngalan, pang-uri, pandiwa, pang-abay at panghalip na nasa anyong paari ang kasunod na salita ay may ang dapat na gamitin. 


$\begin{array}{ll}\text { Korpus } & \text { Deskripsyon } \\ \text { 5. Magandang gabi bayan, } & \text { At pagkalito sa gamit ng mula } \\ \text { mula sa Nobyembre aalisin na } & \text { at simula Itinututuring na } \\ \text { po ang Window Hours ng } & \text { pagkakamali ang labis na } \\ \text { number coding scheme sa Edsa } & \text { pag-uulit ng salita, pasulat man } \\ \text { po at sa C-5 so ibig sabihin po } & \text { ito o pasalita. Ang ikalawang } \\ \text { nito hindi na pwede bumiyahe } & \text { beses na pag-uulit para } \\ \text { ang mga naka-coding sa araw } & \text { bigyang empasis ang isang } \\ \text { na yon sa Edsa at C-5 mula } & \text { salita ay pinapayagan sa } \\ \text { alas-siyete ng umaga } & \text { pagbabalita subalit hindi ang } \\ \text { hanggang alas-siyete ng gabi, } & \text { mga salitang pangkayarian. } \\ \text { nagpapatrol si Jing Castaneda. } & \text { Ang mula ay } \\ & \text { nangangahulugang buhat o } \\ & \text { pinanggalingan; ang simula ay } \\ & \text { tumutukoy sa unang hakbang } \\ & \text { ng anumang gawain o } \\ & \text { panukala o kaya ay unang araw } \\ & \text { o oras ng paggawa. }\end{array}$

6. Ayon sa MERALCO mas higit na dose sentimos kada

kilowatt hour ang bawas singil, ito po'y katumbas ng bente kwatro pesos na bawas singil sa mga kumokonsumo ng two hundred kilowatt per hour kada buwan po iyan.

7. Nakikita na ngayon pa lamang ni Defense Secretary Delfin Lorenzana na magkakaproblema ang sandatahang lakas kung matutuloy na kumuha ng mga China.

Pagkakamali sa redandansi ng mas at higit Ang salitang mas ay nangangahulugang higit o lalo. Ginagamit ito sa paghahambing ng dalawang tao, bagay o pangyayaring

magkaiba at palaging sinusundan ng isang pang-uri na nasa kaantasang lantay.

\section{Modipikasyon}

Magandang gabi bayan, simula sa Nobyembre aalisin na po ang Window Hours ng number coding scheme sa edsa at sa C-5 so ibig sabihin po nito hindi na puwede bumiyahe ang mga naka-coding sa araw na 'yon sa Edsa at C-5 simula alas-siyete ng umaga hanggang alas-siyete ng gabi nagpapatrol si Jing Castaneda. gamit pangdigma sa Russia at

Pagkakamali sa paggamit ng Nakikita na ngayon pa lamang alomorp na pang- Pang, pam at ni Defense Secretary Delfin pang. Batay sa impluwensya ng Lorenzana na katabing tunog, nagkakaroon magkakaproblema ang ng asimilasyon o pagbabago sandatahang lakas kung ang mga alomorp. Ang / $\mathrm{n} /$ - ng matutuloy na kumuha ng mga ay maaaring maging $/ \mathrm{m} / \mathrm{o} / \mathrm{n} /$. gamit pandigma sa Russia at

/m/- labal na $p$ at $b / n /-$ dental China. na $d, l, r, s, t / n /$-gitling + patinig at iba pang katinig ang simula ng salitang -ugat

8. Arestado ang dalawang lalaki Pagkakamali sa paggamit ng 2 dahil sa pangingikil sa isang menor de edad kapalit daw umano $\mathrm{ng}$ hindi pagpapalabas ng sex video sa social media.

salitang may parehong kahulugan -daw at umano. Redandansi Ang daw at umano ay kapuwa nangangahulugan ng sang-ayong sa sabi o narinig. Pagsasabi itong muli ng isang pahayag mula sa ibang tao na ginagamit sa pagpapasa ng balita

9. Naniniwala naman ang mga kaanak ng biktima, na ang mga drug lord sa lugar ang may utak sa krimen.

Pagkakamali sa adisyon ng ang at may May utak vs utak May utak- marunong, maalam Utakmastermind, pu:no ng katiwalian at krimen
Ayon sa MERALCO higit sa dose sentimos kada kilowatt hour ang bawas singil, ito po'y katumbas ng bente kwatro pesos na bawas singil sa mga kumokonsumo ng two hundred kilowatt per hour, kada buwan po iyan.

Arestado ang dalawang lalaki dahil sa pangingikil sa isang menor de edad kapalit daw/umano (alin lang sa dalawa) ng hindi pagpapalabas ng sex video sa social media.

Naniniwala naman ang mga kaanak ng biktima, na mga drug lord sa lugar ang utak sa krimen 
Korpus

10. Sa ilan pang balita, muling hihilingin ng kampo naman $n$ Mark Anthony Fernandez na ilipat ang aktor sa Pampanga Provincial Jail.

\section{Deskripsyon}

Pagkakamali sa paggamit ng ilan pa at iba pa; at pagkakasunod-sunod ng mga salita sa pahayag Sa ilan pang balita- higit sa isang paksa ng balita ang tinutukoy. Maaari itong gamitin sa paghahalayhay ng ulo ng mga pangunahing balita. Sa iba pang balitasegway sa panibago at tiyak na tapik na ibabalita. -Pagtabihin ang mga ingklitik sa pahayag

11. Arestado sa isang buy- bust operation ang isang MMDA traffic enforcer na kung saan tinuturong s'yang supplier ng shabu sa mga driver naman ng pampublikong sasakyan.

12. Natamaan naman si $\mathrm{PO}_{3}$ Julius Albao sa kaliwang kamay kaya itinakbo sa ospital.

Pagkakamali sa paggamit ng salitang kung saan Ang kung saan ay maaaring gamitin sa lugar o bagay na pinagmulan subalit hindi laan para sa tao at sa halip ay iminumungkahing gamitin ang na. Ang na ay isang relatibong (pamanggit) panghalip na katumbas ng who sa ingles. Ang panghalip na pamanggit ay tumutukoy sa pangngalang katabi nito sa pabalik na anyo ng pahayag.

Pagkakamali sa omisyon ng salitang bala bilang tagatanggap ng layon Nawala ang layong ng pandiwang tinamaan. Ipinapayong gumamit ng aktibong pandiwa

13. Full force ang buong cast ng Pagkakamali sa paggamit ng Ang Probinsyano sa kanilang star straded thanks giving concert kagabi. dalawang magkaparehong salita na full force at buo. May redandansi.

14. Sa kabila po ng mga batikos sa kanyang mga maaanghang na mga pahayag at madugong giyera contra illegal drugs, nagpapatuloy pong mataas ang tiwala ng publiko kay Pangulong Digong, yan po'y ayon sa pinakabagong survey ng SWS.

15. Humina ang kalakalan ng ating bansa at China ng dahil nga sa usapin sa West Philippine Sea sa nakaraang mga taon pero dahil sa nakatakdang pagdalaw ni Pangulong Digong sa China, inaasahang lalakas muli ang kalakalan ng dalawang bansa. ang lagyan pa ng pamparaming
Pagkakamali sa paggamit ng dobleng pluralisasyon Ang pang-uring maaanghang ay maramihan na, kalabisan na mga.

Pagkakamali sa paggamit ng sa Humina ang kalakalan ng at nang Sa ang gamitin sa pagtukoy ng petsa o panahon, na nasa aspektong kontemplatibo tulad ng sa linggo, sa 1 ng Disyembre subalit kung natapos na ang kilos o gawa, di na angkop gamitan ng sa, at sa halip ay nang kanyang maaanghang na contra illegal drugs, tiwala ng publiko kay ng SWS.
Modipikasyon

Sa iba pang balita, muli namang hihilingin ng kampo ni Mark Anthony Fernandez na ilipat ang aktor sa Pampanga Provincial Jail.

Arestado sa isang buy- bust operation ang isang MMDA traffic enforcer na itinuturong siyang supplier ng shabu sa mga driver naman ng pampublikong sasakyan.

Tinamaan naman ng bala $\mathrm{si}$ $\mathrm{PO}_{3}$ Julius Albao sa kaliwang kamay kaya itinakbo sa ospital.

Full force/buo (alin sa dalawa) ang cast ng Ang Probinsyano sa kanilang star straded thanks giving concert kagabi.

Sa kabila po ng mga batikos sa pahayag at madugong giyera

gagpapatuloy pong mataas ang Pangulong Digong, yan po'y ayon sa pinakabagong survey pilipinas at ng China ng dahil sa usapin sa West Philippine Sea nang nakaraang mga taon pero dahil sa nakatakdang pagdalaw ni Pangulong Digong sa China, inaasahang lalakas muli ang kalakalan ng dalawang bansa. 
Korpus

16. Puspusan ang

imbestigasyon ngayon ng PNP

sa mga natukoy nilang ninja

cops, isinasailalim na rin sa

lifestyle check ang mga

tiwaling pulis.

17. Jackie kamusta na ang lagay Paggamit ng salitang

ng trapiko sa iyong

kinalalagyan ngayon?

\section{Deskripsyon}

Paglalapit ng pamparaming

mga sa pangngalang

binibigyang turing nito.
Modipikasyon

Puspusan ang imbestigasyon ngayon ng PNP sa natukoy nilang mga ninja cops,

isinasailalim na rin sa lifestyle check ang tiwaling mga pulis.

Jackie kumusta na ang lagay ng trapiko sa iyong kinalalagyan posilisadong kamustanakasanayan subalit hindi iyon ang estandard Kumusta at di kamusta -ang salitang kumusta ay mula sa salitang espanyol na como esta. Kumusta ito sa Filipino at di kamusta

18. Stranded ang libo-libong pasahero sa mga pantalan sa buong kabikulan dahil sa Bagyong Karen at live mula sa Tabacco Port Albay.

19.Samantala itinaas na po ang signal number 1 sa Metro Manila, dahilan nga sa Bagyong pagtukoy ng kinaroroonan at Karen yan ngayon, ang alamin patutunguhan ng bagyo, kundi natin kay kuya kim kung ano ang latest tungkol ho sa Bagyong Karen, Kuya Kim nasan na si Karen, ito ba'y lumakas ba o nagbago ang kanyang takbo o ano? direksiyon, samantalang, ang
20. Hinambalos ng Bagyong Karen ang Catanduanes at iba pang parte ng Southern Luzon.

Pagkakamali sa pagpiling salita Hinagupit ng Bagyong Karen Ang hinagupit at paghampas ay ang Katanduanes at iba pang jargon sa pagbabalita ng panahon na tumutukoy sa hagupit at hampas ng hangin at di hambalos. Magkakaugnay man ang 3 salita ay hindi ginagamit ang hinambalos para ilarawan ang galaw ng hangin.

Pagkakamali sa pagkadinig parte ng Southern Luzon. sunog sa Makati kahapon.
21.Apat na po ang ang umano'y patay sa sunog sa Makati kahapon. dahil sa ingklitik na po Upang maiwasan ang ganitong maling pagkaunawa, iminumungkahing huwag ng gumamit ng po sa mga bilang na may pu sa dulo.

22. Ayon sa warden ito po'y nagsimula dahil sa direktibang ihiwalay ang mga inmates sa kabilang dorm na may kasong kinalaman sa illegal drugs.

Ligaw na panuring (misplaced modifier). llagay ang panuring malapit sa salitang binibigyang turing nito
Pagkakamali sa adisyon ng salitang buo sa kabikulan Pag sinabing kabikulan, tumututukoy ito sa paikot o kabuuan/kalahatan ng Bikol samakatwid hindi na ito kailangang lagyan ng panuring na buo. Pagkakamali sa pagpili ng salita Hindi takbo ang ginagamit sa pagbilis o paghina naman nito ay tinatawag na paggalaw ng bagyo.

Stranded ang libo-libong pasahero sa mga pantalan sa kabikulan dahil sa Bagyong Karen at live mula sa Tabacco Port Albay.

Samantala itinaas na po ang signal number 1 sa Metro Manila, dahilan nga sa Bagyong Karen yan ngayon, ang alamin natin kay Kuya Kim kung ano ang latest tungkol ho sa Bagyong Karen, Kuya Kim nasan na si Karen, ito ba'y lumakas pa o nagbago ang kanyang direksyon o ano?

Apat na ang umano'y patay sa nagsimula dahil sa direktibang ihiwalay ang mga inmate na may kasong kinalaman sa illegal drugs sa kabilang dorm. 


Korpus
23. Bumigat ang daloy ng
trapiko sa South Bound ng $C_{5}$
Road Corner, Pasig Boulevard
kaninang umaga dahil sa isang
truck na may kargang bigas na
sumampa sa Center Island.
Ayon sa Pasig Command Center
nawalan ng control ang driver.
nawalan ng control ang driver.

Pinagbabaril hanggang sa mapatay ng riding-in-tandem ang dalawang barker ng jeep sa Sitio Masagana, Alabang, inaalam pa ng pulis, ang motibo sa pagpatay.
Deskripsyon

Pagkakamali sa paggamit ng salitang nakasanayan ng gamitin o iyong tinatawag na posilisasyon -aalisin na ang na ito ng salitang trapiko. Ang sumampa sa Center Island.

trapiko ay nangangahulugang Ayon sa Pasig Command Center kilos o daloy ng mga nagyayaot nawalan ng control ang driver. (naglalakbay) na tao man o sasakyan sa kalye. At hindi naman bolyum ng sasakyan ang tinutukoy, kundi ang daloy nito.

Pagkakamali sa omisyon ng pamparaming mga pulisindibiduwal; alagad ng batas pulisya- entity; sangay na tagapamayapa kapulisankalahatan

25. Ayon po naman sa pangulo Pagkakamali sa paggamit ng ng senado na si Koko Pimentel, pang-ukol na patungkol sa; at hindi daw kailangang aksyunan gamit ng daw-raw Magkakaiba ang lahat ng mga pahayag patungkol sa senado, ayon sa kanya hindi makasisira sa imahe ng senado ang mga pahayag ni Vice Mayor Paolo Duterte.

26. Ga-hita pa rin ang baha sa mga karatig bayan ng Pampanga.

27. Ayon sa kanila wala po sanang nangyaring gulo kundi unang nanakit ang mga militanteng grupo.

\section{ang ibig sabihin ng mga} salitang patungkol sa, tungkol sa at ukol sa. Ang patungkol sa ay tumutukoy sa anumang bagay na ginagawa bilang pag-alala o pag-aalay sa isang tao (in memory of); ang tungkol sa ay hinggil sa (about); ukol sa ay tulad ng para sa (for). Wastong gamit ng daw/din/; raw/rin. Gamitin ang daw/din, kapag ang sinundang salita ay nagtatapos ra, re, ri, ro,ru at raw, ray, at sa mga katinig, maliban sa malapatinig na w at y; raw/rin naman kung nagtatapos sa patinig at malapatinig.

Pagkakamali sa wastong gami ng panlaping ga- Wastong gamit ng panlaping "ga-" at "abot". Ang panlaping ga- ay ginagamit para ilarawan ang laki (size) ng isang bagay samantalang ang abot- ay tumutukoy naman sa taas nito.

Pagkakamali sa paggamit ng Ayon sa kanila wala po sanang salitang kundi Kundi at Kungdi. nangyaring gulo kungdi unang Ang kundi ay nangangahulugan nanakit ang militanteng mga ng kung at maliban (but grupo. /except); ang kungdi ay pinaikling kung hindi (if not).
Pinagbabaril hanggang sa mapatay ng riding-in-tandem ang dalawang barker ng jeep sa Sitio Masagana, Alabang, inaalam pa ng mga pulis/ng pulisya, ang motibo ng/sa pagpatay.

Ayon po naman sa pangulo ng senado na si Koko Pimentel, hindi raw kailangang aksyunan ang lahat ng mga pahayag tungkol sa senado, ayon sa kanya hindi makasisira sa imahen ng senado ang mga pahayag ni Vice Mayor Paolo Duterte. * siyokoy (alien) ang salitang imahe- ang tama talaga ay imahen dahil ang salita ay mula s salitang Espanyol na imagen. May -n sa dulong bahagi

Abot- hita pa rin ang baha sa mga karatig bayan ng Pampanga. 


\begin{tabular}{|c|c|c|}
\hline Korpus & Deskripsyon & Modipikasyon \\
\hline $\begin{array}{l}\text { 28.Naniniwala naman ang mga } \\
\text { kaanak ng biktima, na ang mga } \\
\text { drug lord sa lugar ang } \\
\text { may-utak sa krimen. }\end{array}$ & $\begin{array}{l}\text { Pagkakamali sa pagpili ng } \\
\text { salitang may- utak. May- utak } \\
\text { at Ang utak. Ang may-utak ay } \\
\text { may kahulugang marunong o } \\
\text { maalam; ang utak ay } \\
\text { tumutukoy sa pasimuno, } \\
\text { pangunahing nag-isip, } \\
\text { mastermind o puno ng } \\
\text { katiwalian }\end{array}$ & $\begin{array}{l}\text { Naniniwala naman ang mga } \\
\text { kaanak ng biktima, na ang mga } \\
\text { drug lord sa lugar ang utak sa } \\
\text { krimen. }\end{array}$ \\
\hline $\begin{array}{l}\text { 29. Patuloy ang imbestigasyon } \\
\text { ng mga otoridad sa nangyaring } \\
\text { pagsabog sa tindahan ng } \\
\text { paputok sa Bocaue, Bulacan } \\
\text { kahapon. }\end{array}$ & $\begin{array}{l}\text { Pagkakamali sa paggamit ng } \\
\text { salitang otoridad Awtoridad at } \\
\text { di otoridad Ang awtoridad ay } \\
\text { mula sa salitang Espanyol na } \\
\text { autoridad. Ang a, e, at o ay } \\
\text { malalakas na patinig, at } \\
\text { maaaring panatilihin ang } \\
\text { baybay nito sa Espanyol kung } \\
\text { ang pantig ay kumbinasyon ng } \\
\text { mga tunog na ito, tulad ng } \\
\text { teorya at oarta. Mahina ang } \\
\text { tunog ng u sa unang pantig sa } \\
\text { au - sa autoridad, kaya't } \\
\text { mapapalitan ito ng w. } \\
\text { Samakatwid, ang basa/bigkas } \\
\text { sa salita ay awtoridad at hindi } \\
\text { otoridad. Ang otoridad ay } \\
\text { salitang siyokoy para sa } \\
\text { awtoridad at bunga ito ng } \\
\text { interlinguwal na pagkakamali } \\
\text { dahil sa kombinasyon o } \\
\text { haluang bigkas Ingles- } \\
\text { Espanyol. Kaparis din ito sa } \\
\text { paraan ng pagbigkas ng } \\
\text { salitang unibersidad na } \\
\text { binibigkas ng marami na } \\
\text { yunibersidad, kalahating } \\
\text { Ingles, kalahating Espanyol. }\end{array}$ & $\begin{array}{l}\text { Patuloy ang imbestigasyon ng } \\
\text { mga awtoridad sa nangyaring } \\
\text { pagsabog sa tindahan ng } \\
\text { paputok sa Bocaue, Bulacan } \\
\text { kahapon }\end{array}$ \\
\hline $\begin{array}{l}\text { 30.Samantala tinanggal na po } \\
\text { sa pwesto ang ilang pulis na } \\
\text { nasasangkot sa marahas na } \\
\text { dispersal ng kilos protesta sa } \\
\text { harap ng us embassy kahapon. } \\
\text { Aminado ang PNP na tila may } \\
\text { pagkukulang din ang kanilang } \\
\text { hanay. }\end{array}$ & $\begin{array}{l}\text { Pagkakamali sa pagpili ng } \\
\text { salitang nasasangkot -Ang } \\
\text { sangkot ay pangangalan na } \\
\text { tumutukoy sa taong dawit, } \\
\text { kasama, at napatunayan ng } \\
\text { kasama sa krimen; ang } \\
\text { nasasangkot na allegedly } \\
\text { involve ay pinaghihinalaan pa } \\
\text { lamang. }\end{array}$ & $\begin{array}{l}\text { Samantala tinanggal na sa } \\
\text { pwesto ang ilang pulis na } \\
\text { sangkot sa marahas na } \\
\text { dispersal ng kilos protesta sa } \\
\text { harap ng us embassy kahapon. } \\
\text { Aminado ang PNP na tila may } \\
\text { pagkukulang din ang kanilang } \\
\text { hanay. }\end{array}$ \\
\hline
\end{tabular}

\subsection{Findings and discussions}

\section{Result of the Analysis}

The study determined that the common errors in the use of Filipino language in TV Newscasting were: 
1. Double pluralization

2. Redundancy

3. Misplaced modifier

4. Omission of modifier due to simplification or automaticity

5. Borrowing of words (salitang siyokoy) that are either Spanish or English

6. Intralingual error or confusing two grammatical rules of two different languages

7. Fossilization or using words that are commonly mistaken because of over literalization.

8. Using enclitic (ingklitik) or terms such as ba, tila, $p a$, and others, and excessive use of $p o$ that is only inserted in sentences that causes confusion instead of clarity.

9. Confusion in words that are almost identical at first glance, but totally different in use and meaning

10. Consecutively using words that have opposite meanings.

11. Pronunciation of words that have identical meanings.

\title{
6. Established Language Rules
}

From the errors that were analysed in the descriptive taxonomy, the following Filipino language rules in writing news script for broadcast media were created. There were more than 100 rules created, and here are some.

\subsection{Language rules in filipino based on the analysis according to its category}

\subsubsection{Addition category}

TABLE 2: Addition Category.

\begin{abstract}
1. Vague automaticity in news casting. The object of the verb needs to be identified correctly to make the news clearer.

2. Parallelism in creating a statement. News reporting is balanced and just, and it needs to be true, not just about its message but also in the process of creating it. If something is used in the first statement, the other statement should also have it for it to be balanced or parallel. Parallelism should be practiced even to content words and function words.
\end{abstract}




\subsection{Omission category}

TABLE 3: Omission Category.

3. Fossilization in the statement Heavy Traffic. Traffic originally means the vehicles, pedestrians, ships, or planes moving along a route that are either slow or fast; in short, it pertains to the flow or movement of the vehicles so we do not have to mention the word flow because it is already encapsulated in the word traffic. Also, the word heavy traffic (bibigat ang trapiko) is loosely and literally translated in Filipino. Congestion is the more appropriate term.

4. Repetition of words with the same meaning to emphasize its message. Repetition of words in news reporting is allowed for emphasis but it should be applied to content words and not to function words. Po, even if it is a part of the Filipino culture, should not be used too much, even articles (ingklitik) because they can hinder the faster relay of message. If possible, remove them from the news reporting.

5. Using Collective Nouns. These kinds of words do not need quantifiers that mean collectively because collective nouns already have this meaning.

6. Redundancy. The word mas in Filipino means more; using this word with another comparative adjectives mean redundancy. All the adjectives or adverbs in the comparative degree do not need additional words that also mean to compare. For example, in English, more weaker is an incorrect term.

7. Using collective nouns meant for two entities. A married couple pertains to two people; if you say two married couples that will pertain to four people. In news casting, we need to avoid dangling modifiers.

\subsection{Correct usage category}

\section{TABLE 4: Correct Usage Category.}

8. The use of acronyms and initials that are not popular to the people (mnemonics) does not allow in news casting. Some exceptions, which the people already know, are allowed such as EJK, USA, UP, SSS, PNP and the like. Its better to use General Advance Planning than GAP.

9. Usage of sa and $\mathrm{ng} . \mathrm{Ng}$ is used when the object of the verb answers the question what, who and which, and sa is used to answer the question where. There are some exceptions, however, in which using them interchangeably is fine. E.g. Ipinatawag siya ng/sa senado. The word senado may represent either the entity or the senatorial group.

10. Usage of daw/din, row/rin. If the preceeding word ends with a consonant and ra, re, ri, ro, ru row and ray, except for semi-vowels W and Y, use dow/din. On the other hand, raw/rin are used if the preceding word ends in a vowel or semi-vowel.

11. Usage of where in (kung saan). The word kung saan is used to pertain to places or entities of starting point, not to people. It is suggested that na and kung siyo be used when pertaining to people. There are several relative pronouns in English- who, which, whom; in Filipino, no is the only equivalent of these.

12. Usage of may and mayroon. Remember that mayroon is used if the next word is a nominative / personal pronoun or enclitic. In usual cases, may is used because most words that it follows are nouns, pronouns in other forms, verbs, adjectives and adverbs.

13. Patungkol sa, tungkol sa and $u k o l$ sa have different meanings. Patungkol sa (in memory of) means anything that is done in honoring or remembering a person; tungkol sa means about; ukol sa means for.

14. Proper usage of kundi and kungdi. The word kundi simply means but or except. Kungdi is the shortened form of kung hindi, which means if not. 
Korpus

Deskripsyon

Modipikasyon

15. Proper usage of the affixes ga- and abot. The affix ga- is used to describe the size of something while abot- describes the height.

\subsection{Word choice category}

TABLE 5: Word Choice Category.

16. Usage of mula and simula. Mula means from; simula means starting point or starting time.

17. Kulungan or bilangguan and bilibid. The word kulungan or bilangguan means jail and is run by city or provincial government. There we can find people who have minor or simple cases.

Bilibid, on the other hand, means prison, handling people with serious cases and is run by the national government.

18. Labi and mga labi. Labi in English is equivalent to remains; that is why it is translated to mgo labi in Filipino. It is a form of fossilization, but still labi is the correct form when pertaining to the remains of one person.

19. Usage of pulis, pulisya and kapulisan. The word pulis is used when pertaining to one policeman; kapulisan is used when pertaining to a group of policemen; pulisya or police means entity or branch of the government that aids in maintaining peace and order in the country.

20. Direksyon and takbo ng Bagyo (typhoon). The words direksyon (way) and kilos or galow (movement), not takbo (status) are usually used to pertain to the typhoon itself. Takbo is the movement itself, probably pertaining to its strength; Direksyon, on the other hand, pertains to the typhoon's track or destination.

21. May- utak at Ang utak. Ang may-utak means intelligent, smart, and bright; ang utak means mastermind of a crime.

22. Sangkot and Nasasangkot. The word sangkot simply means accomplice, a person proven to be associated with another in a crime or a wrong doing, while nasasangkot is a person who is just suspected of being associated with another.

23. /hi.rap/ and/hirap/. The word /hi.rap/ with a slow pronounciation means difficulty, sacrifice, or failure. The word /hirap/, which is pronounced fast, means tired.

24. Awtoridad, not otoridad. The word awtoridad came from the Spanish word autoridad. The loud vowels a, e, and o can maintain its spelling if the syllable is composed of those vowels, like teorya and oarta. The vowel $\mathrm{U}$ is not loud in the first syllable $\mathrm{AU}$ in autoridad. The word otoridad is a result of an interlingual error because of the combined pronounciation of English and Spanish. Another example of this is the word unibersidad (university), pronounced by some as yunibersidad, half English, half Spanish.

\subsection{Proper word order category}

TABle 6: Proper Word Order Category.

25. Placement of markers. Place the markers, pluralizer (mga), articles correctly to the nouns they modify.

26. Misplaced Modifier. Put the modifier in the correct position near the noun it modifies.

\section{Recommendations}

1. Eradicate fossilization in language that is not appropriate in news broadcasting to totally stop common usage mistakes. 
2. Make the students, instructors, experts in Filipino language and practitioners in the field read the created language rules to make this study more useful and productive.

3. Many rules that were created cannot be found in the present national orthography nor in other language manuals, therefore, is recommended to be considered in regard to standardization and intellectualization of Filipino Language.

I am Maria S. Bulaong, director of Sentro ng Wika at Kultura, Region III, also the current dean of the College of Arts and Letters, Bulacan State University; a PH.D. student of Pagpaplanong Pangwika, University of the Philippines, Diliman. Your comments and suggestions are very much welcome. Just text or email me. (0923-594-1467/ mary_bulaong@ rocketmail.com. Thank you very much!

\section{References}

[1] Almario, V. S. and Kilates, M. L., translator 2015. Pagpaplanong wika at Filipino. Manila; Komisyon sa Wikang Filipino

[2] _. Ed. 2014. KWF Manwal sa masinop na pagsulat. Maynila; Komisyon sa Wikang Filipino

[3] Corder, S. P. 1991. Error analysis and interlanguage. USA; Oxford University Press

[4] Dulay, Burt (1977), Krashen (19882). Handbook of research on promoting cross cultural competence

[5] Fairclough, N. 1986. Language and globalization. USA; Raoutledge.

[6] Manwal ng Ortograpiyang Pambansa 2014 ng Komisyon sa Wikang Filipino.

[7] Diskyunaryong Filipino- English 2000 ng Komisyon sa Wikang Filipino

[8] WIKApedia: Balarila at Aralin sa Filipino, 2015 Edisyon. Manila; Presidential Communication Development and Strategic Planning office 